\title{
КОХЛЕАРНАЯ ИМПЛАНТАЦИЯ У НОВОРОЖДЕННЫХ: СЛОЖНОСТИ И ОСОБЕННОСТИ
}

\section{SYSTEMS OF THE COCHLEAR IMPLANTATION IN NEWBORNS: DIFFICULTIES AND FEATURES}

\section{A. Egorov \\ E. Glukhovskiy}

Summary. Hearing loss in each reason cause is always an irreparable loss. It is also an interdiction for adequate socialization and the full development of the talents of a child. Hearing is not only a sensory component of the human body but a trigger mechanism for the development of communication abilities, so, socialization of a person.

Nowadays the cochlear implantation method is the only way to rehabilitate people with hearing loss, and children with deep sensorineural hearing loss. So, it becomes an integrating mechanism into socially and active life.

Setting up a cochlear implant is an important and integral part of the rehabilitation for people with hearing impairments. But in infants, it is not possible to adjust the implants using subjective methods. So, the purpose of the presented research was to study objective options for adjusting a cochlear implant in children under 2 years of age. The presented principles may also be applied to patients without contact in or in the case of comorbidities present in the anamnesis. The authors have assessed the stapedial reflex due to achieve the maximum comfort level for the patient have been stimulated with a cochlear implant. The manuscript also discusses the procedure for measuring auditory reflexes, as well as conducting telemetry of the neural response and the principles of direct measurement of neural responses generated by auditory nerve fibers.

Keywords: cochlear implant, stapedial reflex, neural response telemetry, ECAP.

\section{Вступление}

$\Pi$ отеря слуха - наиболее частая сенсорная депривация. Стоимость лечения глухоты до момента приобретения ребенком речевых и языковых способностей (примерно до 3 лет), в США превышает 1 миллион долларов на ребенка [1]. Число больных с нарушением слуха в Российской Федерации превышает 13 млн. человек, более 1 млн.- дети. Врожденная тугоухость может быть вызвана наследственными, ненаследственными генетическими факторами, осложнениями во время беременности и родов. Наиболее
Егоров Алексей Игоревич

К.ф.-м.н., Московский физико-технический

институm

Глуховский Евгений Михайлович

М.н.с., Московский физико-технический институт glukh.al@myrambler.ru

Аннотация. Потеря слуха по тем или иным причинам всегда невосполнимая утрата, а в отношении ребёнка — это ещё и табу на адекватную социализацию и полноценное развитие талантов ребенка. Слух - это не только сенсорная составляющая функционирования человеческого организма, это пусковой механизм развития коммуникационных способностей, а значит социализации человека в обществе.

На сегодняшний день метод кохлеарной имплантации - это единственный способ реабилитации людей, столкнувшихся с потерей слуха, в частности детей с глубокой нейросенсорной тугоухостью, а значит - интеграции их в социально активную жизнь.

Настройка кохлеарного импланта — важный и неотъемлемый этап реабилитации людей с нарушениями слуховой функции. Но у младенцев проведение настройки имплантов с помощью субъективных методов невозможно. Именно поэтому целью нашей работы стало исследование объективных вариантов настройки кохлеарного импланта у детей до 2 лет. Эти принципы могут также быть применимыми к пациентам, не идущим на контакт, либо же имеющим в анамнезе ряд сопутствующих заболеваний. В статье авторами проведена оценка стапедиального рефлекса с целью достижения максимального уровня комфорта для пациента при стимуляции кохлеарным имплантом. Также в статье рассмотрена процедура измерения слуховых рефлексов, а также — проведение телеметрии нервного ответа и принципы прямого измерения нейронных реакций, генерируемых слуховыми нервными волокнами.

Ключевые слова: кохлеарный имплант, стапедиальний рефлекс, телеметрия нервного ответа, ЕСАР.

распространенные причины потери слуха - это краснуха, сифилис, аномалии развития внутреннего уха; низкая масса тела при рождении; асфиксия в родах; медикаментозная интоксикация ототоксическими препаратами во время беременности; тяжелая желтуха в неонатальном периоде, ведущая к поражению слухового нерва новорожденного [2]. Разделяют кондуктивную и нейросенсорную потерю слуха. Кондуктивная потеря слуха возникает в результате заболеваний наружного слухового прохода и среднего уха. Нейросенсорная тугоухость возникает во внутреннем ухе или вдоль акустических нервных путей [3]. 
На сегодняшний день метод кохлеарной имплантации (КИ) является единственным эффективным способом реабилитации людей с глубокой нейросенсорной тугоухостью. Проведённая операция в раннем возрасте способствует не только приобретению слуховой функции, но и формированию речи [4]. Таким образом, кохлеарная имплантация - это, возможно, единственный шанс для глухого малыша максимально приблизиться к нормально слышащим сверстникам [5].

\section{Важность раннего имплантирования}

Проведение имплантации в возрасте от 6 до 18 мес. обеспечивает начало формирования речи от 1 до 4 мес. после активации устройства [7]. Ранний доступ к звуку через кохлеарный имплантат максимизирует навыки слушания и усвоения языка глухими младенцами в возрасте до 12 месяцев. Частота серьезных и незначительных осложнений сопоставима с исследованиями у взрослых и детей старшего возраста [8-9]. Напротив, глухие дети, которые получили кохлеарный имплант после 7 лет, обычно не могут полноценно овладеть разговорным языком: длительные периоды глухоты приводят к морфологическим изменениям в слуховых областях мозга [10]. Несмотря на очевидные преимущества раннего имплантирования, на практике часто возникает ряд сложностей: позднее выявление потери слуха, медленную оценку степени глухоты, задержки со стороны родителей, осложнения при раннем хирургическом вмешательстве, отсутствие возмещения расходов на медицинское страхование $[6,11]$. Ребенку может быть назначена кохлеарная имплантация, если у него:

- Двусторонняя сенсоневральная потеря слуха с порогами слуха на частотах 500-4000 Гц более 90 дБ;

- Пороги слуха в оптимально подобранном слуховом аппарате более 50 дБ, разборчивость односложных слов менее 20\%;

- Нет противопоказаний к проведению операции под общим наркозом.

- Нет психических и грубых неврологических нарушений, затрудняющих использование КИ и проведение слухоречевой реабилитации;

- Есть возможность регулярно заниматься с сурдопедагогом и родителями после операции.

\section{Принципы работы кохлеарных имплантов}

Кохлеарные импланты (КИ) - это класс нервных протезов, которые могут выполнять роль волосковых клетки улитки, преобразующих механическую энергию в электрические сигналы. Внутреннее устройство кохлеарного имплантата состоит из матрицы внутрикохлеарных электродов и электронного блока, который включает в себя катушку для передачи данных, трансмиссию, генератор импульсов тока и приемник данных. Внешнее устройство кохлеарного имплантата состоит из микрофона для приема звуковых сигналов, сигнального процессора для обработки данных и передающей катушки [12-13].

Сначала звуки воспринимаются микрофоном, затем сигнал от микрофона поступает к речевому процессору. Речевой процессор преобразует звуки в закодированный сигнал, который состоит из последовательности электрических импульсов, который, в свою очередь, передается по кабелю в передатчик и далее в виде радиосигналов через кожу головы к приемнику под кожей. Имплантированный приемник декодирует сигнал и посылает его в виде электрических сигналов на электроды в улитке. Слабые электрические сигналы, передаваемые электродами, стимулируют слуховой нерв. В ответ слуховой нерв передает нервные импульсы мозгу, который воспринимает их как звуки и речь.

\section{Анестезия при проведении кохлеарной имплантации}

Выбор метода анестезии может оказывать влияние на результаты интраоперационного тестирования КИ: чем выше уровень седации пациента, тем меньше средние значения промежуточных точек при регистрации ESRT на различных порогах. Поэтому ряд авторов делают акцент на том, что тестирование КИ нужно проводить на фоне мозговой активности пациента, максимально соответствующей физиологической (показатели BIS-индекса выше 70). Идеальная анестезиологическая техника для имплантации та, которая обеспечивает хорошие условия хирургам и не влияет на измеряемые вызванные слуховые ответы, к примеру, инфузия фентанила и пропофола с кислородом и закисью азота [14-15].

\section{Правильность постановки импланта}

Для наилучшего результата КИ крайне важно оптимальное размещение электродов. Внутрикохлеарная локализация электродов оказывает значительное влияние на распознавание речи и хирургический исход. Следует отметить, что частота неправильного интраоперационного позиционирования электродов достигает 17-33\%. Послеоперационная оценка электродной решетки, позволяющая подтвердить правильное положения электрода, может быть выполнена с помощью рентгенографии, но остается вопрос диагностирования патологий анатомию внутреннего уха. Это может быть решено с помощью компьютерной томографии [16]. 


\section{Настройка процессора}

Контроль качества настроек процессора системы КИ проводится с использованием объективных и субъективных методов. Объективные методы - это регистрация данных без использования ответа ребенка: телеметрия нервного ответа, регистрация стапедиального рефлекса и коротколатентные стволовых потенциалов в ответ на электрическую стимуляцию. К субъективным методам относят детекцию реакции на звук, категоризацию звуков по громкости, аудиометрию в свободном звуковом поле. Только благодаря совместным усилиям специалиста по настройке, педагога и близких ребенка удается правильно настроить параметры речевого процессора и научить ребенка слушать с помощью кохлеарного импланта, а впоследствии - говорить.

В связи с тем, что проведение субъективных методов у младенцев невозможно, целью нашей работы является исследование объективных вариантов настройки кохлеарного импланта у детей до 2 лет.

\section{Результаты и обсужление}

\section{Стапедиальный рефлекс (eSR)}

Хорошо известна корреляция порога стапедиального рефлекса и максимального уровня комфорта для понимания речи. Измерения порога стременного рефлекса, вызванного электрическим током, во время операции могут дать приблизительную информацию, что поможет в начальной настройке речевого процессора кохлеарного имплантата [17].

Одним из наиболее важных шагов для хорошей работы пользователя с КИ является активация и программирование, нацеленные на определение динамического диапазона: между величиной тока (порог для электрического раздражителя) и максимальной интенсивностью электрического раздражения, не вызывающей дискомфорта у пациента (С-уровень). Использование объективных показателей в процессе адаптации КИ способствовало определению динамического диапазона, что показывает хорошую корреляцию с послеоперационными уровнями С и могут обеспечить адекватную помощь в настройке речевого процессора устройства. К тому же, использование данных объективных тестов, как в случае ESRT, делает программирование и отображение электродов КИ более быстрыми и безопасными [18].

Стапедиальный рефлекс - это нормальный вегетативный защитный рефлекс, который защищает ухо от громкого шума. Он состоит из полисинаптической нейронной организации, включающей слуховой нерв в качестве афферентной конечности, а также эфферентный двигательный нейрон, проходящий по лицевому нерву к стременной мышце. Чтобы предотвратить чрезмерную стимуляцию, измеренные во время операции рефлексы стремени могут быть использованы в качестве маркера уровня дискомфорта пациента.

У людей с нормальным слухом стапедиальный рефлекс запускается при уровне звукового давления от 70 до 90 дБ SPL и приводит к сокращению стремени и последующему движению сухожилия стремени. Во время КИ стапедиальный рефлекс запускается принудительно. Движение сухожилия визуализируется под хирургическим микроскопом. Предварительные попытки измерить eSR и определить порог электрически вызванного рефлекса стремени еSRT во время операции выполняется с помощью тимпанометрии контралатеральной стороны и с использованием записей электромиографии стремени. Однако результаты тимпанометрии зависят от морфологии и патологии среднего уха и обычно не воспроизводятся. Кроме того, могут возникнуть трудности с правильным позиционированием зонда в наружном слуховом проходе. Следовательно, этот метод может непропорционально увеличить время операции и вызвать дополнительную хирургическую травму. Использование мер eSRT для установки процессора кохлеарного имплантата является не только более объективным методом, но также является безопасной процедурой для детей и может улучшить распознавание речи $[19,20,25]$.

eSRT - это объективная мера, аналогичная пороговым значениям акустического рефлекса, при котором статическая проводимость слухового прохода (а точнее сокращение стремительной мышцы) отслеживается в ответ на различное количество входного звука, поступающего через КИ. Но приблизительно у 20-30\% пациентов eSRT невозможно измерить. Отсутствию eSRT способствует повреждение стремени во время операции [26-27], аномальные тимпанограммы у пациента, поражение лицевого нерва, неспособность сидеть на месте или непереносимость уровня стимулирующего тона, необходимого для записи ответа. Следует отметить, согласно опросу практикующих сурдологов, еSRT редко используется [28]. И этому есть ряд обьяснений.

\section{eSRT: оборудование, установка и процедура}

eSRT измеряется путем мониторинга изменений статической проводимости слухового прохода, что может указывать на усиление жесткости цепи слуховых косточек в ответ на электрическую стимуляцию. При записи eSRT основным требованием является использование длинного окна записи с целью отслеживания изменения статической проводимости в течение стимуляции. 
Таким образом, клинически сокращение стременной мышцы можно контролировать с помощью теста евстахиевой трубы.

Для измерения eSRT необходимы внешние компоненты КИ (звуковой процессор, кабель), компьютер с программным обеспечением производителя КИ, кабели для программирования КИ, мост иммитанса с протоколом распада рефлекса. Если иммитансный мост должен соединяться с компютером, дополнительно понадобятся кабели, программное обеспечение производителя иммитансного моста, наконечник зонда моста в соответствии с размером уха.

Звуковой процессор КИ будет размещен на пациенте, а звуковой процессор - на ухе или теле в зависимости от стиля звукового процессора. Регистрирующий зонд, прикрепленный к мосту иммитанса, вставляется в ухо пациента. Пороги акустического рефлекса могут различаться примерно на 5 дБ между ипсилатеральными и контралатеральными измерениями. Независимо от размещения датчика, ипсилатерального или контралатерального, протокол распада рефлекса на иммитансном мосту должен быть настроен на контралатеральный ответ. В ухо помещается только корпус датчика. Клиницист может выбрать мониторинг проводников слухового прохода на самом иммитансном мостике, если таковой имеется. Одним из преимуществ записей $\mathrm{eSRT}$ является то, что измерения могут и должны быть получены с использованием тех же параметров стимула в программном обеспечении КИ, которые будут использоваться для поведенческих исследований. Записи eSRT могут быть получены с использованием специфической для канала стимуляции (например, представление тональной посылки с использованием двухфазных импульсов) или для групп электродов (например, стимулы речевой вспышки), в зависимости от о производителе устройства. Способность вызывать eSRT с тем же стимулом, который используется для поведенческой оценки громкости, может быть одной из причин их высокого согласия с поведенческой оценкой громкости.

Необходимым условием для измерения акустических рефлексов является нормально функционирующая система среднего уха. Соответственно, подвижность среднего уха должна быть оценена с помощью тимпанограммы до начала измерения eSRT. Поскольку записи еSRT являются мерой изменения статической проводимости слухового прохода, она чувствительна к движениям пациента: во время процедуры пациент должен оставаться неподвижным и спокойным. Тест eSRT / угасание рефлекса позволит клиницисту использовать иммитансный мост для наблюдения за изменениями статической проводимости слухового прохода.
Чтобы наблюдать это изменение статической проводимости, запись в иммитансный мост должен быть запущена до инициирования стимула в программном обеспечении КИ. Необходимо соблюдать стабильную базовую проводимость всё окно записи. Стабильная базовая линия необходима как эталон для оценки отклонения статической проводимости. Отклонение, представляющее реакцию eSRT, будет отклонением от базовой линии, визуализируемым либо на самом мосту иммитанса, либо в программном обеспечении моста иммитанса, которое привязано по времени к началу и смещению электрической стимуляции, предусмотренной в программном обеспечении производителя КИ.

Важно отметить, что стабильная базовая линия (относительно плоская статическая проводимость) может не опускаться до 0 мл в окне записи. Изменения текущего уровня слуховой системы достигаются посредством манипуляций в программном обеспечении КИ. Другими словами, синхронизированное по времени отклонение eSRT должно изменяться по амплитуде относительно изменений уровня стимуляции в программном обеспечении КИ. Таким образом, увеличение уровня стимуляции в программном обеспечении КИ должно приводить к увеличению амплитуды отклонения eSRT; уменьшение уровня стимуляции в программном обеспечении КИ должно приводить к уменьшению амплитуды отклонения eSRT. Учитывая взаимосвязь между $\mathrm{eSRT}$ и поведенчески измеренными уровнями, разумный уровень представления для начала измерения ESRT будет на комфортном уровне стимулирующего электрода пациента [29].

Уровень стимуляции в программном обеспечении КИ на пороге (отклонение 0,02 на иммитансном мосту) будет регистрироваться для стимулирующего электрода. Одинаковая громкость может быть воспринята, когда верхние уровни стимуляции отражают показатели eSRT. Специфичное для электродов программирование верхних уровней стимуляции приводит к улучшению результатов у пользователей КИ.

\section{Применение ESRT к программированию КИ}

Уровни ESRT могут помочь в установлении верхних уровней электрического динамического диапазона. Люди с потерей слуха могут испытывать трудности с оценкой громкости или описанием сигнала, который они получают от своего имплантата. В этих случаях приходит на помощь измерение уровней eSRT. Tак же, измерения eSRT могут использоваться в качестве перекрестной проверки поведенческих оценок громкости. Использование комбинации еSRT и поведенческих мер может быть особенно полезно при программировании 
детей или трудных для тестирования пациентов [30]. Однако, eSRT не может быть измерен для людей со стимуляцией лицевого нерва или неврологическими нарушениями. Таким образом, аудиолог должен руководствоваться своим клиническим суждением и вносить соответствующие изменения в каждом конкретном случае [21].

\section{Телеметрия нервного ответа}

Среди объективных методов, которые могут быть использованы в программировании речевых процессоров КИ, самым важным является определение порога электрически вызванного потенциала действия (ЭПД) слухового нерва методом телеметрии нервного ответа (ТНО). Уже более 10 лет метод ТНО обеспечивает прямой доступ к регистрации ЭПД и является предметом интереса многих ученых и клиницистов во всем мире. Пороги ТНО определяются двумя способами: визуально и путем экстраполяции данных при регистрации функции зависимости амплитуды ответа ТНО от уровня стимуляции. Выявлена достоверная корреляция профиля ЭПД с профилями ТНО, что позволяет в настоящее время активно использовать эти данные в клинической работе, в особенности в случаях имплантации у детей раннего возраста [22].

Другой метод - это электрически вызванный составной потенциал действия (ЕСАР), измеряемый через устройство после размещения. Хотя ЕСАР изначально использовался для помощи в программировании (он коррелирует с порогами обнаружения и в некоторой степени поведенческими реакциями), он также может предоставить информацию о размещении электродов. В общем, ЕСАР оценивает реакцию слуховой системы пациента на электрическую стимуляцию после введения электрода КИ. Наличие нормальных ответов позволяет хирургу быть уверенным, что все электроды находятся в улитке и функционируют. Он также может предоставить информацию о нефункционирующих электродах или неисправности устройства во время операции, может помочь с начальной активацией устройства, предоставляя пороговые значения для первоначального создания МАР. Это особенно полезно для работы с новорождёнными.

Связь между интраоперационной ТНО и послеоперационными результатами для реципиентов кохлеарных имплантатов сохраняется. Большинство хирургов используют интраоперационную ТНО для детской кохлеарной имплантации в качестве дополнительной меры (для обеспечения правильного размещения электродов и во многих случаях улучшения начальной активации устройства). Интраоперационная ТНО может служить удобным, быстрым способом, если маркер размещения электродов внутри улитки и функционального устройства несовершенный [23].

Программирование детей с кохлеарными имплантатами - это непрерывный постепенный процесс, на который влияют когнитивные способности ребенка, предшествующий слуховой опыт и способность сотрудничать во время длительных сеансов. Следует отметить, что карты детей, которые не могут быть подготовлены к игровой аудиометрии, должны периодически проверяться до тех пор, пока надежные пороги и некоторая шкала громкости не будут получены. Порог ЕСАР используется чаще всего для настройки речевого процессора, особенно у детей с ограниченным слуховым опытом. [24].

\section{Электрически вызванный составной потенциал действия}

Электрически вызванный составной потенциал действия (еСАР) представляет собой синхронизированный ответ группой электрически активируемых волокон слухового нерва. Современные КИ включают в себя возможность «обратной» телеметрии, которая позволяет записывать еСАР в ближнем поле с помощью интракохлеарных электродов. еСАР предлагает ряд преимуществ. Во-первых, для измерения у пациентов с КИ не требуется дополнительное оборудование, специальное программное обеспечение или внешний записывающий электрод. Во-вторых, требует минимального сотрудничества со стороны пациента и не зависит от состояния возбуждения, что является важным преимуществом при работе с детьми. ЕСАР - это прямое измерение нейронных реакций, генерируемых слуховыми нервными волокнами, что делает возможным оценивать исключительно физиологическое состояние слухового нерва [31-33].

\section{Принцип работы ECAP}

ECAР, записанный с использованием интраулиткового электрода у людей, использующих КИ, обычно имеет двухфазную морфологию и состоит из одного отрицательного пика (N1) в пределах временного окна 0,2-0,4 мс после начала стимула и положительного пика (P2), возникающего в районе 0,6-0,8 мс. На этот однопиковый еСАР приходится более $80 \%$ всех измеримых еСАР. Так же, существуют еСАР с двумя положительными пиками (P1 и Р2) — «двойной пик» или «нервный ответ типа Il». Для этого типа ответа еСАР P1 обычно происходит около 0,4-0,5 мс, а Р2 - около 0,6-0,7 мс. Частота ответа типа II составляет около 10-20\%. Различия в задержке между этими двумя пиками могут отражать время распространения спаек по дендриту или по телу спиральных ганглиозных клеток. Амплитуда еСАР может дости- 
гать 1-2 мВ, благодаря чему сохраняется относительно устойчивость к загрязнению и миогенной активности. Особенно ценно, что морфологические характеристики еСАР практически не меняются по мере увеличения продолжительности использования КИ. Но амплитуда еСАР может возрастать по мере увеличения уровня стимуляции. Скорость увеличения можно количественно оценить по наклону функции ввода-вывода еСАР. Кроме того, еСАР, записанные на апикальных электродах, как правило, имеют большую амплитуду, чем записанные на базальных электродах при одинаковом уровне стимуляции или громкости. По мере увеличения расстояния между стимулирующими и перекодирующими электродами задержка еСАР может уменьшаться из-за потенциальных изменений в месте инициирования потенциала действия [34-39].

По сравнению с нормальными слушателями у пользователей КИ ухудшается спектральное разрешение. Электрический ток, подаваемый каждым электродом КИ, создает электрическое поле, которое стимулирует окружающую нервную ткань. Электрические поля, создаваемые разными электродами, обычно перекрываются друг с другом, что приводит к взаимодействию каналов. Отсутствие межволоконной независимости уменьшает количество «эффективных спектральных каналов» многоканального КИ, что ста- вит под угрозу восприятие речи имплантированным пациентам [40-43].

\section{Выво $\Delta ы$}

Настройка речевого процессора у дутей до 2 лет может происходить с помощью только объективных методов. eSRT - полезный инструмент для настройки импланта. Включение измерений eSRT, как части протокола программирования КИ, может помочь аудиологам удовлетворить растущие потребности в создании более эффективных методов программирования КИ, повысить уверенность точности программирования и создать индивидуальные оптимальные параметры имплантируемого устройства.

\section{Б^агоАарности}

Данная статья подготовлена при проведении НИОКТР по теме «Разработка технологии изготовления и постановка на производство импортозамещающей многофункциональной системы кохлеарной имплантации для реабилитации пациентов с сенсоневральной тугоухостью» на базе «Лаборатории медицинского приборостроения» МФТИ. Финансовая поддержка осуществлялась Министерством науки и высшего образования Российской Федерации.

\section{ЛИТЕРАТУРА}

1. Semenov Y.R. Age-Dependent Cost-Utility of Pediatric Cochlear Implantation / Y.R. Semenov, S.T. Yeh, M. Seshamani [et al.] // Ear and Hearing, 2013.—V Vol. 34, Iss. 4, P. 402-412, doi:10.1097/aud.0b013e3182772c66

2. Кузовков В.Е. Приобретенная патология внутреннего уха как фактор, осложняющий хирургический этап кохлеарной имплантации / В.Е. Кузовков, А.С. Лиленко, С.Б. Сугарова, И.В. Костевич // Consilium Medicum, 2018. - Т. 20, № 3, С. 80-84.

3. Brand Y. Cochlear implantation in children and adults in Switzerland / Y. Brand, P. Senn, M. Kompis [et al.] // Swiss Med Wkly, 2014.— Vol. 144, P. w13909, doi:10.4414/smw.2014.13909

4. Королёва И.В. Кохлеарная имплантация у детей младшего возраста / И.В. Королёва, О.С. Жукова, О.В. Зонтова // Новости оториноларингологии и логопатологии, 2002. — № 1, С. 12-24.

5. Семенова К.О. Кохлеарная имплантация как средство помощи глухим детям / К.О. Семенова // Международный журнал гуманитарных и естественных наук, 2016.-Т. 7, № 1, С. 38-42.

6. Исламбекова 3.И. Кохлеарный имплант и его отличие от слухового аппарата / З.И. Исламбекова, Ш. Тахирова // Современное образование (Узбекистан), 2015. - № 12, С. 74-79.

7. Schauwers K. Cochlear implantation between 5 and 20 months of age: the onset of babbling and the audiologic outcome / K. Schauwers, S. Gillis, K. Daemers, C. De Beukelaer, P. Govaerts // J Otol Neurotol, 2004. - Vol. 25, Iss. 3, P. 263-270.

8. Miyamoto R.T. Cochlear implantation in infants below 12 months of age / R.T. Miymoto, B. Colson, S. Henning, D. Pisoni // World Journal of Otorhinolaryngology. Head and Neck Surgery, 2017.—Vol. 3, Iss. 4, P. 214-218, doi: 10.1016/j.wjorl.2017.12.001.

9. Roland J.T. Cochlear implantation in the very young child: Long-term safety and efficacy / J.T. Roland, M. Cosetti, K.H. Wang, S. Immerman, S.B. Waltzman // The Laryngoscope, 2009. — Vol. 119, Iss. 11, P. 2205-2210, doi: 10.1002/lary.20489.

10. Colletti L. Infants versus older children fitted with cochlear implants: Performance over 10 years / L. Colletti, M. Mandalà, L. Zoccante, R.V. Shannon, V. Colletti // International Journal of Pediatric Otorhinolaryngology, 2011. — Vol. 75, Iss. 4, P. 504-509, doi: 10.1016/j.ijporl.2011.01.005

11. Lester E.B. Barriers to the early cochlear implantation of deaf children E.B. Lester, J.D. Dawson, B.J. Gantz [et al.] // Otol Neurotol, 2011.- Vol. 32, P. 406-412.

12. Gwon T.M. A Polymer Cochlear Electrode Array: Atraumatic Deep Insertion, Tripolar Stimulation, and Long-Term Reliability. Springer, 2018. https://doi.org/1007/978-981-13-0472-9. 
13. Орлова 0.С. Совершенствование медико-педагогической реабилитации глухих детей после кохлеарной имплантации посредством настройки речевого процессора // О.С. Орлова, О.В. Зонтова // Специальное образование, 201).— № 2, Т. 50, С. 74-85.

14. Jana J.J. Effect of total intravenous anaesthesia on intraoperative monitoring of cochlear implant function in paediatric patients / J.J. Jana, N. Vaid, J. Shanbhag // Cochlear Implants Int, 2013. - Vol. 14, Iss. 3, P. 169-173, doi: 10.1179/1754762812Y.00000000012.

15. Калугина М.С. Анестезиологическое обеспечение кохлеарных имплантаций у педиатрических пациентов / М.С. Калугина, А.Е. Александров, Е.А. Алексеева, Ю.Ю. Русецкий // Детская хирургия, 2018. — № 22, Т. 4, С. 188-192.

16. Al Omari A. Accuracy of intraoperative electrophysiological testing in confirming correct cochlear implant electrode positions / A. Al Omari, A. Nuseir, M.B. Ata, L. Khasawneh, A. Alhowary, F. Alzoubi // Cochlear Implants International, 2019. - Vol. 20.6, P. 324-330, doi: 10.1080/14670100.2019.1656904.

17. Pau H.W. How reliable is visual assessment of the electrically elicited stapedius reflex threshold during cochlear implant surgery, compared with tympanometry? / H.W. Pau, K. Ehrt, T. Just, U. Sievert, R. Dahl // Jaryngol Otol, 2011. — Vol. 125, Iss. 3, P. 271-273, doi:10.1017/S0022215110002392.

18. de Andrade K.C.L. The Value of Electrically Evoked Stapedius Reflex in Determining the Maximum Comfort Level of a Cochlear Implant / K.C.L. de Andrade, L.F. Muniz, P.L. [et al.] // J Am Acad Audiol, 2018. — Vol. 29, Iss. 4, P. 292-299, doi: 10.3766/jaaa.16117.

19. Weiss N.M. Automated detection of electrically evoked stapedius reflexes (eSR) during cochlear implantation / N.M. Weiss, A. Óvári, T. Oberhoffner [et al.] // Eur Arch Otorhinolaryngol, 2020. - 2020, P. 1-7, doi: https://doi.org/10.1007/s00405-020-06226-X.

20. Bergeron F. Comparison of eSRTs and comfort levels in users of Digisonic SP cochlear implants / F. Bergeron, M. Hotton // Cochlear Implants International, 2014. - Vol. 16, Iss. 2, P. 110-114, doi 10.1179/1754762814Y.0000000092.

21. Messersmith J. Electrically Evoked Stapedial Reflex Threshold: A Procedure / J. Messersmith, L. Entwisle, A. Stout // Perspectives of the ASHA Special Interest Groups, 2018. - Vol. 3, Iss. 9, P. 4-12, doi. 10.1044/persp3.SIG9.4.

22. Бахшинян В.В. Современные тенденции и перспективы применения метода телеметрии нервного ответа в реабилитации пациентов после кохлеарной имплантации / В.В. Бахшинян // Вестник оториноларингологии, 2014.— № 2, С. 21-25.

23. Almosnino G. Use of Neural Response Telemetry for Pediatric Cochlear Implants: Current Practice G. Almosnino, S. Anne, S.R. Schwartz // Ann Otol Rhinol Laryngol, 2018. - Vol. 127, Iss. 6, P. 367-372, doi: 10.1177/0003489418767692.

24. Telmesani L.M. Electrically evoked compound action potential (ECAP) in cochlear implant children: Changes in auditory nerve response in first year of cochlear implant use / L.M. Telmesani, N.M. Said // Int J Pediatr Otorhinolaryngol, 2016. — Vol. 82, P. 28-33, doi:10.1016/j.ijporl.2015.12.027.

25. Weiss B.G. Objective Method to Determine the Electrically Evoked Stapedius Reflex Threshold During Cochlea Implantation / B.G. Weiss, F. Söchting, M. Bertlich [et al.] // An Otol Neurotol, 2018. — Vol. 39, Iss. 1, P. e5-e11, doi:10.1097/MA0.0000000000001611.

26. Wolfe J. Optimizations for the Electrically-Evoked Stapedial Reflex Threshold Measurement in Cochlear Implant Recipients / J. Wolfe, M. Gilbert, E. Schafer [et al.] // Ear Hear, 2017.—Vol. 38, Iss. 2, P. 255-261, doi: 10.1097/AUD.0000000000000390.

27. Walkowiak A. Evoked stapedius reflex and compound action potential thresholds versus most comfortable loudness level: assessment of their relation for charge-based fitting strategies in implant users / A. Walkowiak, A. Lorens, M. Polak [et al.] // ORL J Otorhinolaryngol Relat Spec, 2011.— Vol. 73, Iss. 4, P. 189-195, doi:10.1159/000326892.

28. Hemmingson C. Cochlear Implant Practice Patterns: The U.S. Trends Pediatric Patients / C. Hemmingson, J.J. Messersmith // J Am Acad Audiol, 2018. — Vol. 29, Iss. 8, P. 722-733, doi:10.3766/jaaa.17011.

29. Plant K. Evaluation of streamlined programming procedures for the Nucleus cochlear implant with the Contour electrode array / K. Plant, M.A. Law, L. Whitford [et al.] // Ear Hear, 2005.—Vol. 26, Iss. 6, P. 651-668, doi: 10.1097/01.aud.0000188201.86799.01.

30. Andrade K.C. The importance of electrically evoked stapedial reflex in cochlear implant/K.C. Andrade, C. Leal Mde, L.F. Muniz, L. [et al.] // Braz J Otorhinolaryngol, 2014. — Vol. 80, Iss. 1, P. 68-77, doi: 10.5935/1808-8694.20140014

31. He S. The Electrically Evoked Compound Action Potential: From Laboratory to Clinic / S. He, H.F.B. Teagle, C.A. Buchman // Front Neurosci, 2017.— Vol. 11, P. 339, doi: 10.3389/fnins.2017.00339.

32. Kim J.R. The relationship between electrically evoked compound action potential and speech perception: a study in cochlear implant users with short electrode array / J.R. Kim, P.J. Abbas, C.J. Brown [et al.] // Otol Neurotol, 2010.—Vol. 31, Iss. 7, P. 1041-1048, doi: 10.1097/MA0.0b013e3181ec1d92.

33. Garadat S.N. Using temporal modulation sensitivity to select stimulation sites for processor MAPs in cochlear implant listeners / S.N. Garadat, T.A. Zwolan, B.E. Pfingst // Audiol Neurootol, 2013.—Vol. 18, Iss. 4, P. 247-260, doi: 10.1159/000351302.

34. Cafarelli Dees D. Normative findings of electrically evoked compound action potential measurements using the neural response telemetry of the Nucleus $\mathrm{CI} 24 \mathrm{M}$ cochlear implant system D. Cafarelli Dees, N. Dillier, W.K. Lai [et al.] // Audiol Neurotol, 2005. — Vol. 10, P. 105-116, doi: 10.1159/000083366.

35. Van de Heyning P. Electrically evoked compound action potentials are different depending on the site of cochlear stimulation / P. Van de Heyning, S.L. Arauz, M. Atlas [et al.] // Cochlear Implants Int, 2016. — Vol. 17, P. 251-262, doi: 10.1080/14670100.2016.1240427

36. Lai W.K. A simple two-component model of the electrically evoked compound action potential in the human cochlea / W.K. Lai, N. Dillier // Audiol Neurotol, 2000. - Vol. 5, P. 333-345, doi: 10.1159/000013899.

37. Kashio A. Exploring the source of neural response of different latencies obtained from different recording electrodes in cochlear implant users / A. Kashio, V.D. Tejani, R.A. Scheperle, C.J. Brown, P.J. Abbas // Audiol Neurotol, 2016. — Vol. 21, P. 141-149, doi: 10.1159/000444739.

38. Baudhuin J.L. A comparison of alternating polarity and forward masking artifact-reduction methods to resolve the electrically evoked compound action potentials / J.L. Baudhuin, M.L. Hughes, J.L. Goehring // Ear Hear, 2016. - Vol. 4, P. 247-255, doi: 10.1097/AUD.0000000000000288.

39. Undurraga J.A. Polarity effects on neural responses of the electrically stimulated auditory nerve at different cochlear sites / J.A. Undurraga, A. van Wieringen, R.P. Carlyon, 0. Macherey, J. Wouters // Hear Res, 2010.—Vol. 269, P. 146-161, doi: 10.1016/j.heares.2010.06.017. 
40. Winn, M. B., and Litovsky, R. Y. (2015). Using speech sounds to test functional spectral resolution in listeners with cochlear implants. J. Acoust. Soc. Am. 137, 1430-1442. doi: 10.1121/1.4908308

41. Winn M.B. Assessment of spectral and temporal resolution in cochlear implant users using psychoacoustic discrimination and speech cue categorization / M.B. Winn, J.H. Won, I.J. Moon // Ear Hear, 2016.—Vol. 37, P. e377-e390, doi: 10.1097/AUD.00000000000000328.

42. Jones G.L. Relationship between channel interaction and spectral-ripple discrimination in cochlear implant users / G.L. Jones, J.H. Won, W.R. Drennan, J.T. Rubinstein // J Acoust Soc Am, 2013. — Vol. 133, P. 425-433, doi: 10.1121/1.4768881.

43. Noble J.H. Image-guidance enables new methods for customizing cochlear implant stimulation strategies / J.H. Noble, R.F. Labadie, R.H. Gifford, B.M. Dawant // IEEE Trans Neural Syst Rehabil Eng, 2013.—Vol. 21, P. 820-829, doi: 10.1109/TNSRE.2013.2253333.

\section{(c) Егоров Алексей Игоревич, Глуховский Евгений Михайлович ( glukh.al@myrambler.ru ).}

Журнал «Современная наука: актуальные проблемы теории и практики»

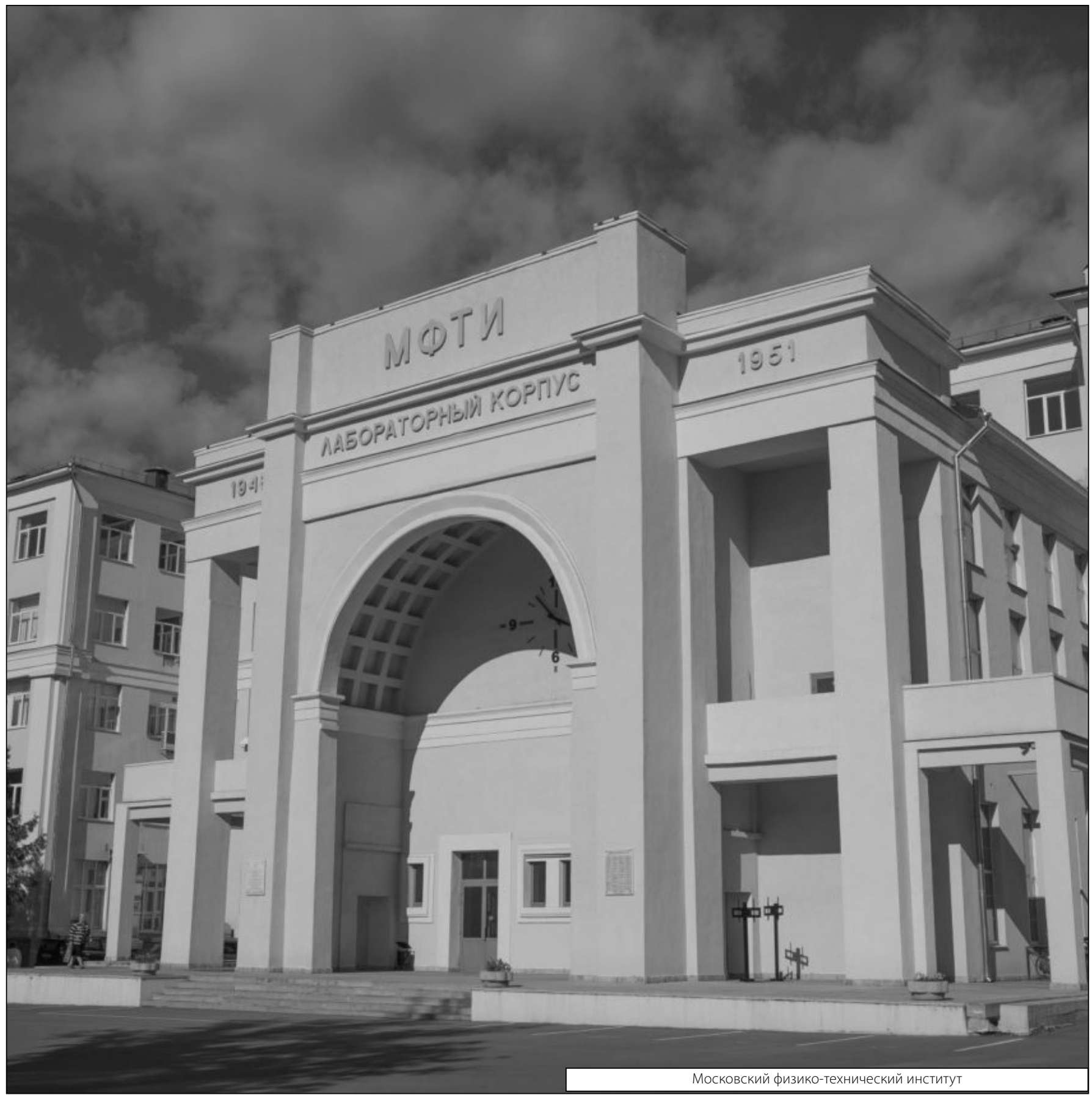

